\title{
Variable stars in the field of the young open cluster Roslund 2
}

\author{
P. Sowicka ${ }^{1}$, G. Handler ${ }^{2}$, R. Taubner ${ }^{3}$, M. Brunner ${ }^{3}$, \\ V.-M. Passegger ${ }^{3}$, F. Bauer ${ }^{3}$, and E. Paunzen ${ }^{4}$ \\ ${ }^{1}$ Astronomical Observatory, Jagiellonian University, Orla 171, 30-244 Kraków, Poland, \\ email: paula@byk.oa.uj.edu.pl \\ ${ }^{2}$ Nicolaus Copernicus Astronomical Center, Bartycka 18, 00-716 Warsaw, Poland \\ ${ }^{3}$ Institut für Astronomie, Universität Wien, Türkenschanzstrasse 17, 1180 Wien, Austria \\ ${ }^{4}$ Department of Theoretical Physics and Astrophysics, Masaryk University, Kotlarska 2, \\ 61137 Brno, Czech Republic
}

\begin{abstract}
The study of variable stars in open clusters via asteroseismology is a powerful tool for the study of stellar evolution and stars in general. That is because stars in clusters can be assumed to originate from the same interstellar cloud, so they share similar properties such as age and overall metallicity. We performed a search for variable stars in the field of the young open star cluster Roslund 2, with photoelectric and CCD photometry acquired at two different telescopes. Within the resulting light curves we have found 12 variable stars. Our measurements confirm three previously known variables.
\end{abstract}

Keywords. open clusters and associations: individual (Roslund 2), stars: variables

\section{Introduction}

The cluster Roslund 2 is a young ( $~ 8 \mathrm{Myr}$, Kharchenko et al. 2005) open cluster, found in a survey of high luminosity stars in the northern Milky Way (Roslund 1960). It is the biggest cluster found in this survey, with a diameter of about $45^{\prime}$. No dedicated search for variable stars in this cluster is available in the literature.

Studying pulsating stars in open clusters has many advantages. Most importantly we can assume the same origin for all stars, i.e. we have tight constraints on their ages, metallicity and distance, which helps in asteroseismic investigations. The B-type pulsators, which naturally occur in open clusters, are good targets for asteroseismology. So it is important to find more such stars in open clusters and that was the purpose of our measurements.

\section{Observations, data processing and searching for variable stars}

The observations were performed using the 0.8-m "vienna little telescope" (vlt) at the Institute of Astronomy of the University of Vienna and the 0.75-m Automatic Photoelectric Telescope (APT) T6 at Fairborn Observatory in Arizona. We used 56 hours of CCD photometry in the $V$ filter (vlt) and 42 hours of data in the Strömgren uvy filters (APT).

The reduction of the vlt data was made using standard procedures. For photometry we used two programs: DAOPHOT/ALLSTAR and DIAPL2 (Difference Image Analysis Package) (W. Pych, private communication). After visual examination of our light curves we found three variables. We cleaned the light curves by rejecting points by $3 \sigma$ level and 

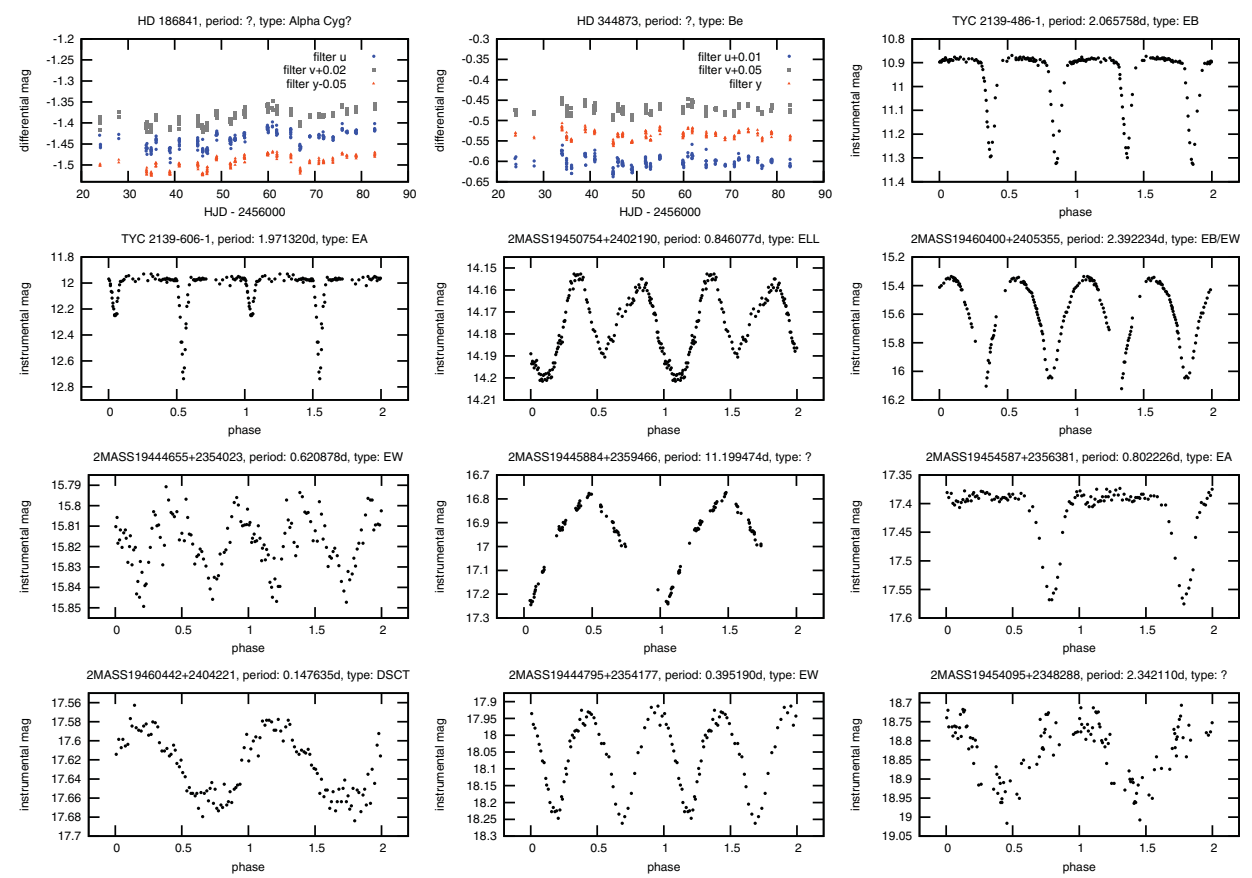

Figure 1. Resulting light curves of the variable stars

we found five additional variable stars. Afterwards we used program PERLARGE to compute Fourier amplitude spectra for all stars, and we found two more variables.

The APT data were reduced following standard photoelectric photometry schemes. The targets were: HD 186841, HD 344870, HD 344873 and HD 344878. Because of a nearby contaminating star, the measurements of HD 344878 were useless. HD 186841 was confirmed to be variable. HD 344870 was most constant and was used as a comparison star. HD 344873 was discovered to be variable.

For most of found variables we determined primary values of periods, based on the nice shape of the phased light curve. Figure 1 shows the resulting light curves, with the values of periods (if determined) and first classifications of the variable star types.

\section{Summary}

We performed a search for variable stars in the field of the young open star cluster Roslund 2. Three of the variables we observed were previously known, and we determined periods for ten stars. Unfortunately, none of these variables is likely to be of $\beta$ Cephei type.

Acknowledgements. We are grateful to Wojtek Pych for permission to use and continued support of his DIAPL2 software. This research has been supported by the Polish NCN grant 2011/01/B/ST9/05448. PS acknowledges the Summer Student Program at the Nicolaus Copernicus Astronomical Center in Warsaw and IAU Grant.

\section{References}

Roslund, C. 1960, PASP, 72, 205

Kharchenko, N. V., Piskunov, A. E., Röser, S., Schilbach, E., \& Scholz, R.-D. 2005, A $\& A, 438$, 1163 